\title{
Intervenção telefônica na prática de autocuidado com os pés em diabéticos: ensaio clínico randomizado*
}

\author{
Telephone intervention in self-care practices with the feet of patients with diabetes: \\ a randomized clinical trial \\ Intervención telefónica en la práctica del autocuidado con los pies en diabéticos: \\ ensayo clínico aleatorizado
}

Como citar este artigo

Silva AFR, Moura KR, Moura TVC, Oliveira ASS, Moreira TMM, Silva ARV. Telephone intervention in self-care practices with the feet of patients with diabetes: a randomized clinical trial. Rev Esc Enferm USP. 2021;55:e03737 . https://doi.org/10.1590/S1980-220X2020047203737

Antonia Fabiana Rodrigues da Silva ${ }^{1}$

(D) Karine Rafaela de Moura ${ }^{2}$

Tatiana Victória Carneiro Moura ${ }^{1}$

Andressa Suelly Saturnino de

Oliveira $^{3}$

Thereza Maria Magalhães Moreira ${ }^{4}$

Ana Roberta Vilarouca da Silva ${ }^{5}$

* Extraído da dissertação "A análise do uso de intervenção telefônica, realizada por enfermeiro, no autocuidado para prevenção do pé diabético”, concluída no Programa

de Pós-Graduação Ciências e Saúde,

Universidade Federal do Piauí, 2020

${ }^{1}$ Universidade Federal do Piauí, Picos, PI, Brasil.

${ }^{2}$ Universidade Estadual do Piauí, Picos, PI, Brasil.

${ }^{3}$ Universidade da Integração Internacional da Lusofonia Afro-Brasileira, Redenção, CE, Brasil.

${ }^{4}$ Universidade Estadual do Ceará, Departamento de Enfermagem, Fortaleza, CE, Brasil.

${ }^{5}$ Universidade Federal do Piauí, Departamento de Enfermagem, Teresina, PI, Brasil.

\section{ABSTRACT}

Objective: To assess the effect of a telephone intervention for self-care practices with the feet of people with type 2 diabetes mellitus. Method: This is a randomized clinical trial with two groups, control and intervention, carried out with 102 users with type 2 diabetes mellitus linked to four Basic Health Units in a municipality in the state of Piauí. For the intervention group, six telephone interventions were carried out in three months; and the control group received only conventional follow-up. For assessment, a form was applied before and after the intervention period. Results: In the intragroup analysis, when comparing self-care practices with the feet in the control group in the preand post-tests, there was no significant difference ( $p>0.05)$; in the intervention group, there was an increase in self-care practices in $70 \%$ of the items, with $p<0.001$ to 0.03 . When analyzing the practice of intergroup self-care after the telephone intervention, the positive effect of the intervention was evidenced, obtaining statistically significant differences in $60 \%$ of the items, with a value of $p<0.001$ to 0.031 . Conclusion: The telephone intervention promoted greater adherence to self-care practices with the feet of patients with type 2 diabetes mellitus. Brazilian Clinical Trials Registry: RBR-3pq5th.

\section{DESCRIPTORS}

Health Education; Diabetic Foot; Diabetes Mellitus; Telephone; Self-care.

Rua Arão José Ferreira, 325, 


\section{INTRODUÇÃO}

"O pé diabético está entre as complicações mais frequentes do diabetes mellitus (DM) e suas consequências podem ser dramáticas para a vida do indivíduo, gerando desde feridas crônicas e infecções até amputações de membros inferiores"(1). "As complicações do pé diabético são responsáveis por $40 \%$ a $70 \%$ do total de amputações não traumáticas de membros inferiores na população geral e $85 \%$ das amputações de membros inferiores em pessoas com DM são precedidas de ulcerações, sendo os seus principais fatores de risco a neuropatia periférica, as deformidades no pé e os traumatismos"(1).

O conhecimento sobre os cuidados adequados com os pés e a prática do autocuidado podem reduzir as alterações prejudiciais que predispõem o surgimento de úlceras e feridas, além de reduzir grande parte das amputações de membros inferiores ${ }^{(2)}$. Com isso, é necessário desenvolver atividades de educação em saúde para melhorar o conhecimento do paciente diabético sobre os cuidados com os pés e estimulá-lo a praticar tais cuidados, em busca de minimizar ou até mesmo prevenir a ocorrência das complicações. Diante disso, surge a necessidade de utilizar novas abordagens e estratégias de suporte para suprir limitações e auxiliar as práticas convencionais de educação em saúde durante as consultas de enfermagem.

Neste cenário inovador, "destacam-se: suporte telefônico para o cuidado em saúde; serviços telefônicos gratuitos de emergência; acompanhamento da adesão ao tratamento; lembretes de compromissos; ações de promoção da saúde; campanhas de educação em saúde”, dentre outros $^{(3)}$. Apesar de o suporte telefônico já ser utilizado na medicina há alguns anos em vários países, ainda é pouco difundido na assistência de enfermagem no Brasil. "A utilização do telefone surge como uma estratégia potencial para o cuidado integral que se traduz em uma expansão da ação em saúde, representando uma evolução frente ao tradicional cuidar" ${ }^{(4)}$.

Além disso, estudos que utilizaram a intervenção telefônica constataram melhora no autocuidado sobre alimentação e na prática de exercício físico ${ }^{(5)}$, impacto positivo na redução dos valores da glico-hemoglobina e na prática de insulinoterapia ${ }^{(6-8)}$. Ressalta-se que essa modalidade não substitui os atendimentos face a face, pois é implementada como suporte, em caráter de complementaridade ao atendimento convencional.

Assim, pressupõe-se que a utilização de intervenções educativas via chamadas telefônicas na sensibilização dos pacientes com DM2, realizadas por enfermeiros, oferecerá subsídios para uma ação educativa que possibilite o aconselhamento e incentivo para maior adesão à prática de autocuidado com os pés, visando tanto a redução de complicações dos pacientes diabéticos quanto a prevenção do surgimento de lesões e amputações futuras. Desta forma, o objetivo deste estudo foi avaliar o efeito de uma intervenção telefônica para a promoção da prática de autocuidado com os pés, em pessoas com DM2, acompanhadas na atenção básica.

\section{MÉTODO}

\section{TIPO DE ESTUDO}

Trata-se de um ensaio clínico randomizado aberto realizado com dois grupos: Grupo Controle (GC) e Grupo Intervenção (GI).

\section{População}

A população-base do estudo foi composta pelos pacientes diagnosticados com DM2, cadastrados em unidades de atenção primária. Foram incluídos usuários com diagnóstico de DM2 há, pelo menos, dois anos, associado ou não à hipertensão arterial, com idade igual ou superior a 18 anos e com acesso à linha de telefone fixo ou aparelho celular. Foram excluídos os que já possuíam o diagnóstico prévio pé diabético, algum déficit cognitivo, auditivo ou de fala, informado pelos participantes/familiar que impossibilitasse resposta direta aos instrumentos de coleta de dados, os que não atenderam as ligações após quatro tentativas consecutivas, bem como os que mudaram de telefone no decorrer do estudo.

A amostra foi calculada a partir da fórmula para estudos com comparação entre grupos ${ }^{(9)}$, colocando-se uma margem de $10 \%$ para eventuais perdas. Verificou-se, assim, que seriam necessárias 64 pessoas em cada grupo, totalizando 128.

\section{Coleta de dados}

A pesquisa foi desenvolvida com os usuários de quatro Unidades Básicas de Saúde (UBS) do município de Picos, Piauí, realizada por uma enfermeira durante o período de dezembro de 2018 a julho de 2019. As UBS foram selecionadas por meio do sorteio via programa computacional e sem reposição.

O estudo ocorreu em três etapas: 1) pré-intervenção (antes do início da intervenção telefônica e da randomização); 2) intervenção educativa; 3) pós-intervenção. $\mathrm{Na}$ primeira etapa, por meio da aplicação de um formulário de modo presencial, foi realizado o primeiro contato com os usuários nas UBS, e foram coletados dados sobre endereço e telefone, variáveis sociodemográficas: sexo, idade, estado civil, escolaridade, moradia e renda familiar; e variáveis clínicas: peso, estatura, pressão arterial (PA), índice de massa corporal (IMC) e prática de autocuidado com pés.

O peso foi obtido por meio de uma balança digital portátil da marca Techline $e^{\circledast}$ com capacidade máxima de $150 \mathrm{~kg}$, e a estatura foi verificada a partir de uma fita antropométrica fixada a uma superfície vertical plana e sem desníveis, com escala entre $1,0 \mathrm{~cm}$ e 2,0 m. O IMC foi obtido a partir da razão entre peso $(\mathrm{kg})$ e quadrado da altura $(\mathrm{m})$. Utilizaram-se os parâmetros específicos para adulto, classificando-os como baixo peso $\left(<18 \mathrm{~kg} / \mathrm{m}^{2}\right)$, peso normal $\left(18,5\right.$ a $\left.24,9 \mathrm{~kg} / \mathrm{m}^{2}\right)$ e excesso de peso $\left(25,0 \text { a } 29,0 \mathrm{~kg} / \mathrm{m}^{2}\right)^{(10)}$; e para idosos, classificando-os como baixo peso $\left(\leq 22 \mathrm{~kg} / \mathrm{m}^{2}\right)$, adequado ou eutrófico $\left(>22 \mathrm{e}<27 \mathrm{~kg} / \mathrm{m}^{2}\right)$ e sobrepeso $\left(\geq 27 \mathrm{~kg} / \mathrm{m}^{2}\right)^{(11)}$.

A verificação da PA foi realizada com esfigmomanômetros aneroides da marca Welch Allyn Tycos $^{\mathrm{ML}}$ e estetoscópios biauriculares da marca Littmann ${ }^{\oplus}$. Classificaram-se como normotensos os participantes que apresentaram Pressão Arterial Sistólica (PAS) < $140 \mathrm{mmHg}$ e Pressão Arterial Diastólica $(\mathrm{PAD})<90 \mathrm{mmHg}^{(12)}$. 
Para a avaliação da prática de autocuidado com os pés, foi utilizado um instrumento, uma adaptação sobre conhecimento e prática de autocuidado de pacientes diabéticos no que concerne à prevenção de lesões nos pés, composta por dez itens, aplicada nas etapas pré e pós-intervenção, sendo eles: lavagem dos pés pelo menos uma vez por dia, seca dos pés após o banho e secagem entre os dedos do pé; hidratação dos pés com cremes ou óleos hidrantes nos calcanhares e na planta do pé (palma do pé ou parte de baixo do pé); uso de meias de algodão; uso de calçado fechado e inspeciona (olha) na parte de dentro antes de calçá-lo; corte das unhas no formato reto; uso de tesoura sem ponta para cortá-las.

O desfecho considerado para este estudo foi a prática de autocuidado com os pés dos usuários diabéticos, medida por meio desses dez itens, em que, após a intervenção, esperava-se uma diferença de $25 \%$ do GI em relação ao GC em mais de $50 \%$ dos itens.

Após o término do recrutamento, os participantes foram randomizados a um dos grupos (GC ou GI), através do Research Randomizer, por um profissional da estatística, gerando grupos verdadeiramente comparáveis e proporcionais (1:1), em que a amostra alocada em cada grupo foi proporcionalmente ajustada, considerando o sexo e o tempo de diagnóstico para DM.

A segunda etapa, a intervenção telefônica, nomeada de programa Ligue-pé, teve duração de três meses, sobre orientações de autocuidado com os pés, ocorrendo de maneira sistematizada junto ao GI pela pesquisadora. O objetivo da intervenção foi fornecer orientações, motivar e encorajar os indivíduos a praticar o autocuidado com os pés. A intervenção ocorreu através de contatos telefônicos, que foram realizados a cada 15 dias, por uma enfermeira treinada, de março a maio de 2019. Foram seis contatos telefônicos por paciente, com média de duração de sete minutos, realizados de segunda a sexta, no horário da manhã, das 8 às 12 h, e no período da tarde, das 14 às $17 \mathrm{~h}$.

Destaca-se que as ligações telefônicas com orientações sobre os cuidados com pés foram realizadas, exclusivamente, pela autora deste estudo, que teve acesso aos registros dos dados de cada participante. As intervenções telefônicas seguiram um roteiro previamente elaborado, com conteúdos a serem abordados em cada contato (Quadro 1). Os conteúdos foram elaborados a partir de informações contidas no Manual do Pé Diabético ${ }^{(1)}$ e no Consenso Internacional do Pé Diabético ${ }^{(13)}$.

Quadro 1 - Conteúdos abordados na intervenção telefônica Picos, PI, Brasil, 2019.

\begin{tabular}{|c|c|}
\hline Intervenção telefônica $(\mathbf{n}=\mathbf{6})$ & Conteúdo da intervenção \\
\hline $1^{\circ}$ contato & Inspeção e autoexame dos pés \\
\hline $2^{\circ}$ contato & Lavagem e secagem dos pés \\
\hline $3^{\circ}$ contato & Hidratação e uso de meias \\
\hline $4^{\circ}$ contato & $\begin{array}{c}\text { Uso de calçados (tipo, formato e } \\
\text { material) }\end{array}$ \\
\hline $5^{\circ}$ contato & Cuidados com unhas e calos \\
\hline $6^{\circ}$ contato & Atividade física e alimentação \\
\hline
\end{tabular}

$\mathrm{Na}$ terceira etapa, pós-intervenção, ocorreu a aplicação do pós-teste 15 dias após as intervenções telefônicas. Nessa etapa, foi promovida a reavaliação da prática de autocuidado mediante a aplicação da quinta parte do formulário sobre conhecimento e prática de autocuidado de pacientes diabéticos no que concerne à prevenção de lesões nos pés, através do contato telefônico.

Ressalta-se que o GI continuou recebendo o atendimento de rotina na UBS, já o GC, recebeu somente o atendimento de rotina na UBS, com orientações que são oferecidas durante as consultas de enfermagem e médicas. Para a aplicação do pós-teste, mesmo o GC não tendo recebido nenhuma intervenção telefônica, foi respeitado o tempo da intervenção do GI. A aplicação do pós-teste, no GC, também ocorreu através de contato telefônico.

Por se tratar de um estudo de intervenção educativa, não foi possível cegar a pesquisadora responsável pela intervenção, pois era necessário entrar em contato por telefone com os participantes para realizar a intervenção, logo, trata-se de um ensaio aberto. Para garantir a confiabilidade na mensuração do desfecho, as enfermeiras e os demais profissionais da UBS não foram informados quais pacientes faziam parte do GI ou GC. Na análise dos dados, por sua vez, foi realizada por um profissional da estatística; também não foi informado para o grupo em que o paciente havia sido alocado.

\section{ANÁLISE E TRATAMENTO DOS DADOS}

Para realizar as análises estatísticas dos dados, foram utilizados os software R, versão 3.5.3, utilizado para os Testes QuiQuadrado de homogeneidade, e o Statistical Package for the Social Sciences (SPSS), versão 20, utilizado para realizar o Teste de McNemar e o Teste $t$ de Student. Para as variáveis qualitativas, utilizaram-se as frequências absolutas (n) e relativas (\%); e para as quantitativas, utilizaram-se média e desvio padrão.

Para comparar a prática de autocuidado com os pés em diabéticos nos tempos antes e após a intervenção, na análise intragrupo, foi utilizado o Teste de McNemar devido à característica de amostragem pareada. Nas comparações intergrupo, foi utilizado o Teste Qui-Quadrado de homogeneidade para comparar proporções. Para as variáveis contínuas, utilizou-se Teste $t$ de Student para amostras independentes com variâncias iguais, e para testar a homogeneidade das variâncias, foi utilizado o Teste de Levene. Para todas as análises, foi considerado um nível de 0,05 de significância.

\section{AspeCtos ÉTIICOS}

$\mathrm{O}$ desenvolvimento do estudo atendeu às normas de ética em pesquisa envolvendo seres humanos, conforme a Resolução no 466/2012 do Conselho Nacional de Saúde $(\mathrm{CNS})^{(14)}$. A pesquisa foi aprovada pelo Comitê de Ética em Pesquisa (CEP) da Universidade Federal do Piauí (UFPI), no ano de 2018, com número de Parecer 3.018.155/2018 e está cadastrada na Plataforma de Registros Brasileiros de Ensaios Clínicos, com cadastro nacional: RBR-3pq5th e número de identificação do UTN: U1111-1218-1886.

Os pacientes foram abordados nas UBS, e, quando eles adentravam a unidade para consultas, receber medicações e marcar exames, eram também encaminhados à sala 
disponibilizada para a coleta. Em seguida, era explicado o objetivo do estudo e questionados sobre o interesse em participar do estudo. Todos os usuários que concordaram em participar assinaram o Termo de Consentimento Livre e Esclarecido (TCLE), em duas vias.

\section{RESULTADOS}

Do total de 128 usuários da amostra, no GI, no primeiro contato telefônico, quatro informaram desistência; não foi possível estabelecer contato telefônico durante a intervenção telefônica com sete (número de telefone não existia ou não atendeu às ligações) e um desenvolveu cegueira; na fase final, em dois participantes, não foi possível aplicar o pós-teste. No GC, não foi possível aplicar o pós-teste em 12 participantes. Desta forma, a amostra final do estudo foi constituída por 102 usuários, sendo 50 pertencentes ao GI e 52 ao GC (Figura 1).

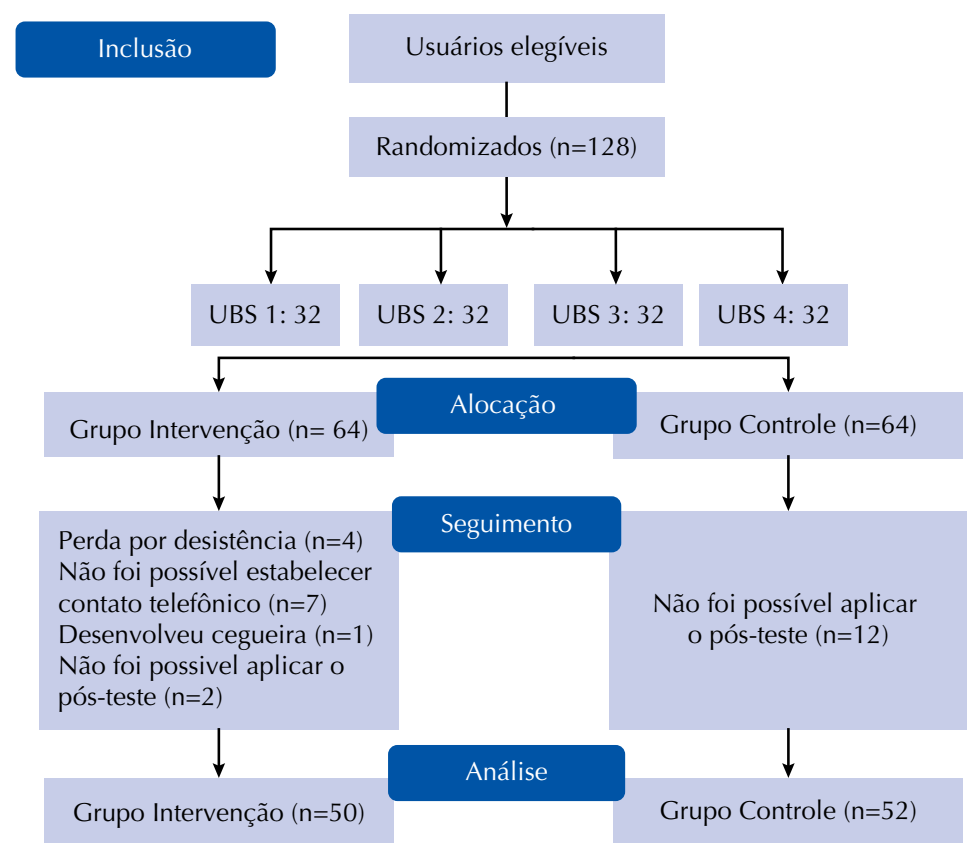

Figura 1 - Diagrama CONSORT: Processo de alocação dos usuários nas fases do ensaio clínico - Picos, PI, Brasil, 2019.

Sobre os dados sociodemográficos, nos 102 (100\%) participantes, a média de idade, em ambos os grupos, foi superior a 60 anos $(\mathrm{GC}=65, \mathrm{GI}=61, \mathrm{p}=0,079)$. A maioria era do sexo feminino $(\mathrm{GC}=73 \%, \mathrm{GI}=68 \%, \mathrm{p}=0,574)$, aposentados $(\mathrm{GC}=67,3 \%, \mathrm{GI}=44 \%, \mathrm{p}=0,019)$ e casa$\operatorname{dos}(\mathrm{GC}=58 \%, \mathrm{GI}=66 \%, \mathrm{p}=0,388)$. Apesar de a maior parte dos usuários do GI possuir ensino fundamental completo (48\%) e a maioria no GC ser analfabetos (49\%), não houve associação estatística $(\mathrm{p}<0,05)$. No que se refere à renda mensal média, no GC, foi de $\mathrm{R} \$ 1.679,7$ e no GI, de $R \$ 1.624,9$, sem diferença significativa $(p=0,759)$. Dessa forma, os grupos são homogêneos segundo as variáveis sociodemográficos, exceto pela ocupação.

No que tange aos dados clínicos, ambos os grupos apresentaram tempo médio de diagnóstico de mais ou menos oito anos, um IMC médio de 26,4 (GC) e 27 (GI) $\mathrm{kg} / \mathrm{m}^{2}$; valores da PAD de 77,6 (GI) e 78,1 (GC) mmHg; PAS de 127,2 (GI) e 130,8 (GC) mmHg. Conforme apresentado na Tabela 1, é possível verificar que os grupos são homogêneos segundo as variáveis clínicas $(\mathrm{p}>0,05)$.

Tabela 1 - Características clínicas dos participantes da pesquisa - Picos, PI, Brasil, 2019

\begin{tabular}{lccc}
\hline Variáveis clínicas & GC $(\mathbf{n}=\mathbf{5 2})$ & Gl $(\mathbf{n}=\mathbf{5 0})$ & Valor de $\mathbf{p} *$ \\
\cline { 2 - 3 } & $\begin{array}{c}\text { Média } \\
\text { (desvio padrão) }\end{array}$ & $\begin{array}{c}\text { Média } \\
\text { (desvio padrão) }\end{array}$ & 0,560 \\
\hline IMC $\left(\mathrm{kg} / \mathrm{m}^{2}\right)$ & $26,4(4,7)$ & $27(4,8)$ & 0,909 \\
Tempo de DM (anos) & $8,33(6,76)$ & $8,5(8,3)$ & 0,821 \\
Pressão Arterial Diastólica & $78,1(10,5)$ & $77,6(10,8)$ & 0,291 \\
Pressão Arterial Sistólica & $130,8(17,7)$ & $127,2(16,2)$ & 0 \\
\hline
\end{tabular}

Nota: *Teste $t$ de Student para amostras independentes. 
Com relação à análise intragrupo no GC, observou-se que não houve diferença significativa $(\mathrm{p}>0,05)$ na prática de autocuidado com os pés, por parte dos pacientes diabéticos, entre os tempos pré e pós-teste. Já ao comparar a prática de autocuidado com os pés, no GI, nos tempos pré e pós-teste, notou-se que, após a intervenção, houve maior adesão de prática de autocuidado em 70\% dos itens avaliados, obtendo-se um valor de $\mathrm{p}<0,001$ a 0,031. Ressalta-se que a prática de lavar os pés já estava consolidada entre os participantes (100\%), portanto, não havia espaços para melhoria no pós-teste (Tabela 2).

Tabela 2 - Prática de autocuidado com os pés, intragrupo, no pré e pós-teste - Picos, PI, Brasil, 2019

\begin{tabular}{|c|c|c|c|c|c|c|}
\hline \multirow[b]{2}{*}{ Variáveis } & \multicolumn{3}{|c|}{ Grupo controle } & \multicolumn{3}{|c|}{ Grupo intervenção } \\
\hline & $\begin{array}{l}\text { Pré-teste } \\
\%\end{array}$ & $\begin{array}{l}\text { Pós-teste } \\
\%\end{array}$ & $\mathbf{p}^{* *}$ & $\begin{array}{l}\text { Pré-teste } \\
\%\end{array}$ & $\begin{array}{l}\text { Pós-teste } \\
\%\end{array}$ & $\mathbf{p}^{* *}$ \\
\hline \multicolumn{7}{|l|}{ Prática de autocuidado com os pés } \\
\hline Lava os pés pelo menos uma vez por dia & 100,0 & 100,0 & 1,000 & 100,0 & 100,0 & 1,000 \\
\hline Seca os pés após o banho & 75,0 & 75,0 & 1,000 & 74,0 & 88,0 & 0,020 \\
\hline Após o banho seca entre os dedos do pé & 40,4 & 40,4 & 1,000 & 34,0 & 84,0 & $<0,001$ \\
\hline $\begin{array}{l}\text { Faz hidratação dos pés com cremes ou } \\
\text { óleos hidratantes nos calcanhares }\end{array}$ & 59,6 & 69,2 & 0,060 & 58,0 & 98,0 & $<0,001$ \\
\hline $\begin{array}{l}\text { Faz hidratação na planta do pé (palma do } \\
\text { pé ou parte de baixo do pé) }\end{array}$ & 34,6 & 21,2 & 0,092 & 44,0 & 64,0 & 0,002 \\
\hline Usa meias de algodão & 26,9 & 28,8 & 1,000 & 36,0 & 32,0 & 0,625 \\
\hline Usa calçado fechado & 48,1 & 48,1 & 1,000 & 44,0 & 40,0 & 0,625 \\
\hline $\begin{array}{l}\text { Inspeciona (olha) todo dia a parte de } \\
\text { dentro antes de calçá-lo }\end{array}$ & 40,4 & 38,5 & 1,000 & 48,0 & 82,0 & $<0,001$ \\
\hline Corta as unhas sempre retas & 25,0 & 23,1 & 1,000 & 20,0 & 72,0 & $<0,001$ \\
\hline Corta as unhas com tesoura sem ponta & 3,8 & 1,9 & 1,000 & 4,0 & 16,0 & 0,031 \\
\hline
\end{tabular}

Nota: ${ }^{* *}$ Teste de McNemar; $\%$ - porcentagem.

Em relação à comparação intergrupal, no pré-teste, sobre a prática de autocuidado com os pés, pode-se observar que não houve diferença estatística indicando homogeneidade na prática de autocuidado entre os grupos. Entre os itens praticados por mais de $50 \%$ dos pesquisados, no pré-teste, em ambos os grupos, foram somente três: lavar os pés ao menos uma vez por dia; secar os pés após o banho; hidratação dos pés com cremes ou óleos hidratantes nos calcanhares. No que se refere à comparação intergrupal após a intervenção telefônica, no pós-teste, nota-se o efeito positivo da intervenção, já que foram obtidas diferenças estatisticamente significativas em $60 \%$ dos itens, com valor de $\mathrm{p}<0,001$ a 0,031 (Tabela 3).

Tabela 3 - Comparação da prática de autocuidado com os pés, intergrupo, entre o GC e GI, no pré e pós-teste - Picos, PI, Brasil, 2019.

\begin{tabular}{|c|c|c|c|c|c|c|}
\hline \multirow{2}{*}{ Variáveis } & \multicolumn{3}{|c|}{ Pré-teste } & \multicolumn{3}{|c|}{ Pós-teste } \\
\hline & GC \% & GI \% & $\mathbf{p}^{* * *}$ & GC $\%$ & GI \% & $\mathbf{p}^{* * *}$ \\
\hline \multicolumn{7}{|l|}{ Prática de autocuidado com os pés } \\
\hline Lava os pés pelo menos uma vez por dia & 100 & 100 & 1,000 & 100,0 & 100,0 & 1,000 \\
\hline Seca os pés após o banho & 75 & 74 & 1,000 & 75,0 & 88,0 & 0,152 \\
\hline Após o banho seca entre os dedos do pé & 40 & 34 & 0,644 & 40,4 & 84,0 & $<0,001$ \\
\hline $\begin{array}{l}\text { Faz hidratação dos pés com cremes ou } \\
\text { óleos hidratantes nos calcanhares }\end{array}$ & 60 & 58 & 1,000 & 69,2 & 98,0 & $<0,001$ \\
\hline $\begin{array}{l}\text { Faz hidratação na planta do pé (palma do } \\
\text { pé ou parte de baixo do pé) }\end{array}$ & 35 & 44 & 0,443 & 21,2 & 64,0 & $<0,001$ \\
\hline Usa meias de algodão & 27 & 36 & 0,439 & 28,8 & 32,0 & 0,896 \\
\hline Usa calçado fechado & 48 & 44 & 0,830 & 48,1 & 40,0 & 0,534 \\
\hline $\begin{array}{l}\text { Inspeciona (olha) todo dia a parte de } \\
\text { dentro antes de calçá-lo }\end{array}$ & 40 & 48 & 0,565 & 38,5 & 82,0 & $<0,001$ \\
\hline Corta as unhas sempre retas & 25 & 20 & 0,714 & 23,1 & 72,0 & $<0,001$ \\
\hline Corta as unhas com tesoura sem ponta & 4 & 4 & 1,000 & 1,9 & 16,0 & 0,031 \\
\hline
\end{tabular}

Nota: ***Teste Qui-Quadrado para proporção. 


\section{DISCUSSÃO}

O estudo avaliou o efeito de uma intervenção educativa via telefone, com enfoque em orientações relacionadas ao autocuidado com os pés, obtendo como resultado um efeito positivo da intervenção, pois os usuários que receberam a intervenção telefônica apresentaram maior adesão à prática de autocuidado com os pés em comparação ao GC.

Com relação ao perfil dos participantes, corroborando os achados de outros estudos realizados com usuários com DM2 em UBS, os resultados demonstram que a maioria dos pacientes é do sexo feminino, possuem companheiro e têm, em média, mais de 60 anos $^{(15-18)}$. O tempo médio de diagnóstico encontrado foi superior a oito anos em ambos os grupos, representando, portanto, um potencial agravante para as possíveis complicações da DM2, apresentando um IMC normal para idosos ${ }^{(15,19)}$.

Com relação aos estudos internacionais voltados para o uso de intervenção telefônica para a prevenção e autocuidado com os pés de pacientes diabéticos, abordaram como tecnologia, diferentemente do presente estudo, o uso de mensagens de texto por telefone celular, para reforçar o aprendizado e a prática de cuidados com os pés de usuários com diabetes; assim como nesse estudo, obtiveram melhores resultados na adesão à prática desses cuidados os que receberam a intervenção ${ }^{(20-21)}$.

Outros estudos realizados com diabéticos apontam resultados satisfatórios com o uso da intervenção telefônica, em que os escores gerais de autocuidado nos grupos de intervenção foram significativamente maiores do que no $\mathrm{GC}^{(22)}$, na adesão à dieta para diabéticos, exercícios, cuidados com os pés, monitoramento da glicemia e uso de medicamentos ${ }^{(23-24)}$.

Sobre os estudos brasileiros que fizeram uso da intervenção telefônica com pacientes diabéticos, observa-se que não eram voltados para reforçar os cuidados com os pés, mas abordaram a intervenção telefônica como capaz de promover uma forma de comunicação efetiva por parte do profissional e da pessoa com DM2, já que as ligações telefônicas possibilitam diálogo, reflexão e maior corresponsabilização da pessoa com condição crônica em relação à própria saúde ${ }^{(16,25)}$.

A maioria dos estudos que fizeram uso de intervenção telefônica com usuários diabéticos obtiveram resultados satisfatórios, em que os participantes que receberam as intervenções apresentaram melhores resultados na adesão à alimentação saudável ${ }^{(25)}$, na promoção do autocuidado relacionado à atividade física e ao seguimento de um plano alimentar ${ }^{(16)}$, na redução dos fatores de risco cardiovascular e a melhoria da qualidade de vida relacionada à saúde ${ }^{(26)}$. Por meio das ligações, é possível fazer recomendações sobre alimentação, atividade física, uso correto de medicamentos, cuidados com os pés e até mesmo ensinar a identificar sintomas de hiperglicemia e hipoglicemia ${ }^{(27)}$.

Alem disso, um estudo observacional, ao avaliar o suporte telefônico como estratégia para o monitoramento do DM, identificou grande satisfação por parte dos usuários com as ligações telefônicas, tendo suas expectativas atendidas ${ }^{(28)}$.
Destaca-se ainda que "o uso do telefone é considerado um método eficaz para abordar o usuário em sua casa ou comunidade, em horários flexíveis, além de otimizar o tempo"(16).

Dessa forma, a telemedicina pode ser um complemento importante no tratamento do usuário com DM2, melhorando a educação do paciente e o conhecimento sobre a doença. "Além disso, ela pode ajudar o sistema de saúde, aliviando a sobrecarga em ambientes de atendimento especializado e apoiando o papel de administrador da atenção primária” ${ }^{(29)}$.

Observa-se que o acompanhamento com o uso de uma abordagem centrada nos usuários, estimulando-os a terem autonomia na realização das práticas de autocuidado, contribuiu para melhorar as práticas de cuidados com os pés entre os usuários do GI. Logo, o contato telefônico se mostrou uma opção de comunicação viável e efetiva para o acompanhamento dos usuários com DM2, por ser simples, cômodo, rápido e de baixo custo. $\mathrm{O}$ telefone encurta as distâncias físicas e socioculturais, fortalecendo o vínculo e facilitando a escuta e compreensão, principalmente quando se trata da Atenção Básica ${ }^{(30)}$.

Durante o planejamento e a execução desta pesquisa, foram considerados alguns aspectos que poderiam influenciar o efeito positivo da intervenção telefônica, como as ligações serem realizadas por somente uma enfermeira, a padronização da linguagem e a qualidade das informações prestadas. Visto isso, elaborou-se um roteiro para orientar as chamadas e organizar os dias e horários das ligações para garantir o sucesso do contato.

No entanto, algumas limitações devem ser pontuadas. Dentre elas estão: o curto período de acompanhamento; a ausência da análise de algumas variáveis clínicas, como a glicemia capilar e a glicohemoglobina; a impossibilidade de cegamento dos participantes do estudo; a diferença na ocupação entre os grupos. No entanto, essas limitações não invalidam os achados desse estudo e podem resultar em desdobramentos para estudos posteriores.

\section{CONCLUSÃO}

A realização da intervenção telefônica, como estratégia educativa, realizada com usuários com DM2 acompanhados na atenção básica, evidenciou maior adesão à prática de autocuidado com os pés no GI em comparação ao GC. Ressalta-se a importância do uso desse tipo de estratégia ser contínua para manter esse resultado satisfatório, bem como ampliar o autocuidado.

Os resultados desta pesquisa mostram os benefícios da intervenção telefônica como uma estratégia inovadora que pode auxiliar na melhora da prática de autocuidado com os pés em pacientes com DM2. Com isso, espera-se que este estudo tenha desdobramentos futuros, podendo implementar a intervenção telefônica como suporte ao tratamento convencional (consultas) utilizado nos serviços de Atenção Básica. Acredita-se que a tendência é de agregar várias estratégias educativas voltadas para estimular a adesão às práticas de autocuidado em DM2 e que essas estratégias possam ser avaliadas quanto à sua contribuição, principalmente nos cuidados com os pés. 


\section{RESUMO}

Objetivo: Avaliar o efeito de uma intervenção telefônica para a prática de autocuidado com os pés em pessoas com diabetes mellitus tipo 2. Método: Ensaio clínico randomizado com dois grupos, controle e intervenção, realizado com 102 usuários com diabetes mellitus tipo 2, vinculados a quatro Unidades Básicas de Saúde de um município do Piauí. Para o grupo intervenção, foram realizadas seis intervenções telefônicas em três meses, e o grupo controle recebeu somente o acompanhamento convencional. Para a avaliação, aplicouse um formulário antes e após o período das intervenções. Resultados: $\mathrm{Na}$ análise intragrupo, ao comparar a prática de autocuidado com os pés no grupo controle, no pré e pós-teste, não houve diferença significativa $(p>0,05)$; já no grupo intervenção, houve aumento da prática de autocuidado em $70 \%$ dos itens, obtendo-se p < 0,001 a 0,03. Ao analisar a prática de autocuidado intergrupal após a intervenção telefônica, evidenciou-se o efeito positivo da intervenção, obtendo diferenças estatisticamente significativas em $60 \%$ dos itens, com valor de $\mathrm{p}<0,001$ a 0,031. Conclusão: A intervenção telefônica promoveu maior adesão à prática de autocuidado com os pés dos pacientes com diabetes mellitus tipo 2. Registro Brasileiro de Ensaios Clínicos: RBR-3pq5th.

\section{DESCRITORES}

Educação em Saúde; Pé Diabético; Diabetes Mellitus; Telefone; Autocuidado.

\section{RESUMEN}

Objetivo: Evaluar el efecto de una intervención telefónica para la práctica del autocuidado con los pies en personas con diabetes mellitus tipo 2. Método: Ensayo clínico aleatorizado con dos grupos, control e intervención, realizado con 102 usuarios con diabetes mellitus tipo 2, vinculado a cuatro Unidades Básicas de Salud en un municipio de Piauí. Para el grupo de intervención, se llevaron a cabo seis intervenciones telefónicas en tres meses y el grupo de control recibió solo un seguimiento convencional. Para la evaluación se aplicó un formulario antes y después del período de intervención. Resultados: En el análisis intragrupo, al comparar la práctica del autocuidado con los pies en el grupo control, en el pre y post test, no hubo diferencia significativa ( $p>0,05)$; en el grupo de intervención, hubo un incremento en la práctica del autocuidado en el $70 \%$ de los ítems, obteniendo $\mathrm{p}<0,001$ a 0,03 . Al analizar la práctica del autocuidado intergrupal posterior a la intervención telefónica, se evidenció el efecto positivo de la intervención, obteniendo diferencias estadísticamente significativas en el $60 \%$ de los ítems, con un valor de $p<0,001$ a 0,031 . Conclusión: La intervención telefónica promovió una mayor adherencia a la práctica del autocuidado con los pies de los pacientes con diabetes mellitus tipo 2 . Registro Brasileño de Ensayos Clínicos: RBR-3pq5th.

\section{DESCRIPTORES}

Educación en Salud; Pie Diabético; Diabetes Mellitus; Teléfono; Autocuidado.

\section{REFERÊNCIAS}

1. Brasil. Ministério da Saúde, Secretaria de Atenção à Saúde. Manual do pé diabético: estratégias para o cuidado da pessoa com doença crônica [Internet]. Brasília, DF: Secretaria de Atenção à Saúde; 2016 [cited 2019 Nov 20]. Available from: http://www.as.saude.ms.gov. br/wp-content/uploads/2016/06/manual_do_pe_diabetico.pdf

2. Policarpo NS, Moura JR, Melo Junior EB, Almeida PC, Macêdo SF, Vilarouca AR. Conhecimento, atitudes e práticas de medidas preventivas sobre pé diabético. Rev Gaúcha Enferm. 2014;35(3):36-42. https://doi.org/10.1590/1983-1447.2014.03.45187

3. Rocha TA, Fachini LA, Thumé E, Silva NC, Barbosa AC, Carmo MD, et al. [Mobile health: new perspectives for healthcare provision]. Epidemiol Serv Saude. 2016;25(1):159-70. Portuguese. https://doi.org/10.5123/S1679-49742016000100016

4. Vasconcelos HC, Freitas RW, Marinho NB, Lima FE, Araújo TL, Damasceno MM. Effectiveness of telephone interventions as a strategy for glycemic control: an integrative literature review. Texto Contexto Enferm. 2013;22(1):239-46. https://doi.org/10.1590/S0104-07072013000100029

5. Sousa Z, Neves C, Carvalho D. [Insulin administration technique: a practice based on scientific evidence]. Rev Port Diabetes [Internet]. 2019 [cited 8 Aug 2020];14(3):120-8. Portuguese. Available from: http://www.revportdiabetes.com/wp-content/uploads/2019/11/RPD-Set2019-Artigo-de-Revis\%C3\%A3o-p\%C3\%A1gs-120-128.pdf

6. Hunt CW. Technology and diabetes self-management: an integrative review. World J Diabetes. 2015;6(2):225-33. https://doi.org/10.4239/ wjd.v6.i2.225

7. Becker TA, Teixeira CR, Zanetti ML, Pace AE, Almeida FA, Torquato MT. Effects of supportive telephone counseling in the metabolic control of elderly people with diabetes mellitus. Rev Bras Enferm. 2017;70(4):704-10. https://doi.org/10.1590/0034-7167-2017-0089

8. Schechter CB, Walker EA, Ortega FM, Chamany S, Silver LD. Costs and effects of a telephonic diabetes self-management support intervention using health educators. J Diabetes Complications. 2016;30(2):300-5. https://doi.org/10.1016/j.jdiacomp.2015.11.017

9. Hulley SB, Cummings SR, Browner WS, Grady DG, Newman TB. Delineando a pesquisa clínica. 4th ed. Porto Alegre: ArtMed; 2015.

10. World Health Organization. Obesity: preventing and managing the global epidemic: Report of a WHO Consultation [Internet]. Geneva: World Health Organization; 2019 [cited 2019 Oct 6]. Available from: https://www.who.int/nutrition/publications/obesity/WHO_TRS_894/en/

11. Lipschitz DA. Screening for nutritional status in the elderly. Prim Care [Internet]. 1994 [cited 2019 Sep 20];21(1):55-67. Available from: https://www.ncbi.nlm.nih.gov/pubmed/8197257

12. Sociedade Brasileira de Cardiologia, Socieade Brasileira de Hipertensão, Sociedade Brasileira de Nefrologia. $7^{\mathrm{a}}$ Diretriz brasileira de hipertensão arterial [internet]. Rio de Janeiro: Sociedade Brasileira de Cardiologia; 2016 [citado 2019 Sept 2]. Available from: https://sbc-portal.s3.sa-east-1.amazonaws.com/diretrizes/Pocket\%20Books/2017/7\%C2\%AA\%20Diretriz\%20Brasileira\%20de\%20 Hipertens $\%$ C3\%A30\%20Arterial.pdf

13. Brasil. Ministério da Saúde. Grupo de Trabalho Internacional sobre Pé Diabético. Consenso internacional sobre pé diabético [Internet]. Brasília, DF: Secretaria do Estado de Saúde do Distrito Federal; 2011 [cited 2019 Jul 2]. Available from: http://189.28.128.100/dab/docs/ publicacoes/geral/conce_inter_pediabetico.pdf

14. Brasil. Ministério da Saúde, Conselho Nacional de Saúde. Resolução № 466, de 12 de dezembro de 2012. Aprova as diretrizes e normas reguladoras de pesquisa envolvendo seres humanos [Internet]. Brasília, DF: Conselho Nacional de Saúde; 2012 [cited 2019 Mar 2]. Available from: https://conselho.saude.gov.br/resolucoes/2012/Reso466.pdf 
15. Marques MB, Coutinho JF, Martins MC, Lopes MV, Maia JC, Silva MJ. Educational intervention to promote self-care in older adults with diabetes mellitus. Rev Esc Enferm USP. 2019;53:e03517. https://doi.org/10.1590/s1980-220x2018026703517

16. Fernandes BS, Reis IA, Torres HC. Evaluation of the telephone intervention in the promotion of diabetes self-care: a randomized clinical trial. Rev Lat Am Enfermagem. 2016;24:e2719. https://doi.org/10.1590/1518-8345.0632.2719

17. Nguyen TP, Edwards H, Do TN, Finlayson K. Effectiveness of a theory-based foot care education program (3STEPFUN) in improving foot self-care behaviours and foot risk factors for ulceration in people with type 2 diabetes. Diabetes Res Clin Pract. 2019;29:e38. https://doi.org/10.1016/j.diabres.2019.05.003

18. Hushie M. Exploring the barriers and facilitators of dietary self-care for type 2 diabetes: a qualitative study in Ghana. Health Promot Perspect. 2019;9(3):223-32. https://doi.org/10.15171/hpp.2019.31

19. Arrelias CA, Faria HT, Teixeira CR, Santos MA, Zanetti ML. Adherence to diabetes mellitus treatment and sociodemographic, clinical and metabolic control variables. Acta Paul Enferm. 2015;28(4):315-22. https://doi.org/10.1590/1982-0194201500054

20. Hassan ZM. Mobile phone text messaging to improve knowledge and practice of diabetic foot care in a developing country: feasibility and outcomes. Int J Nurs Pract. 2017;23 Suppl 1:e12546. https://doi.org/10.1111/ijn.12546

21. Naghibi SA, Moosazadeh M, Zhyanifard A, Jafari Makrani Z, Yazdani Cherati J. Analyzing short message services application effect on diabetic patients' self-caring [Internet]. Int J Prev Med. 2015;6:75. https://doi.org/10.4103/2008-7802.162670

22. Hemmati Maslakpak M, Razmara S, Niazkhani Z. Effects of face-to-face and telephone-based family-oriented education on selfcare behavior and patient outcomes in type 2 Diabetes: a randomized controlled trial. J Diabetes Res. 2017;2017:8404328. https://doi.org/10.1155/2017/8404328

23. Nesari M, Zakerimoghadam M, Rajab A, Bassampour S, Faghihzadeh S. Effect of telephone follow-up on adherence to a diabetes therapeutic regimen. Jpn J Nurs Sci. 2010;7(2):121-8. https://doi.org/10.1111/j.1742-7924.2010.00146.x

24. Razmara Iranagh S, Hemmati Maslakpak M. The effect of family-based telephone follow-up on self-care of patients with Diabetes. J Holist Nurs Midwifery. 2018;28(1):84-91. https://doi.org/10.18869/acadpub.hnmj.28.1.84

25. Franco RC, Becker TAC, Hodniki PP, Zanetti ML, Sigoli, PBO, Teixeira CRS. Telephone support for adherence to healthy eating practices among people with type 2 diabetes mellitus. Enferm Glob. 2018;(50):176-184. https://doi.org/10.6018/eglobal. 17.2.277821

26. Vílchez Barboza V, Klijn TP, Salazar Molina A, Sáez Carrillo KL. Effectiveness of personalized face-to-face and telephone nursing counseling interventions for cardiovascular risk factors: a controlled clinical trial. Rev Lat Am Enfermagem. 2016;24:e2747. https://doi.org/10.1590/1518-8345.0626.2747

27. Johnson M, Jastrzab R, Tate J, Johnson K, Hall-Lipsy E, Martin r, et al. evaluation of an academic-community partnership to implement $\mathrm{mtm}$ services in rural communities to improve pharmaceutical care for patients with Diabetes and/or Hypertension. J Manag Care Spec Pharm. 2018;24(2):132-41. https://doi.org/10.18553/jmcp.2018.24.2.132

28. Olivatto GM, Teixeira CR, Pereira MC, Becker TA, Istilli PT. [ATEMDIMEL - Telephone support for monitoring in diabetes mellitus type 2: expectations and patient satisfaction]. Rev Eletr Gest Saúde. 2015;6(2):1588-600. Portuguese. https://doi.org/10.18673/gs.v6i2.22487

29. Moreira AM, Marobin R, Rados DV, Farias CB, Coelli S, Bernardi BL, et al. Effects of nurse telesupport on transition between specialized and primary care in diabetic patients: study protocol for a randomized controlled trial. Trials. 2017;18(1):222. https://doi.org/10.1186/ s13063-017-1954-z

30. Sousa LO, Figueiredo WS, Machado ML. [The education practices in diabetes experienced in SUS: a literature discussion with emphasis on Primary Health Care]. Rev APS. 2017;20(3):423-33. Portuguese. https://doi.org/10.34019/1809-8363.2017.v20.15801

Coordenação de Aperfeiçoamento de Pessoal de Nível Superior - Brasil (Capes). 